\title{
HEMATOMA EPIDURAL ESPINHAL PÓS-TRAUMÁTICO AGUDO: UM RELATO DE CASO
}

\author{
Acute post-traumatic spinal epidural hematoma: a case report
}

\section{Hugo Nonato Lustosa Correia ${ }^{1}$ D Wendel Vilar Duque da Rosa ${ }^{1}$ Klícia Magalhães Pereira ${ }^{2}$ Jéssica Lima Carvalhido Antônio ${ }^{2}$ (1) Wilson Okabayashi'}

${ }^{1}$ Centro Universitário de Valença (UNIFAA) Valença $(R J)$

${ }^{2}$ Hospital Escola de Valença - Valença (RJ)

Autor correspondente:

Hugo Nonato Lustosa Correia

E-mail: hugolustosa8@gmail.com

\section{Como citar este artigo:}

CORREIA, H.N.L.; ROSA, W.V.D.; PEREIRA, K.M.; ANTÔNIO, J.L.C.; OKABAYASHI, W Hematoma epidural espinhal pós-traumático: um relato de caso. Revista Saber Digital, v. 14, n. 3 , p. 23-29, 2021.

Data de Submissão: 15/11/21

Data de aprovação: $28 / 11 / 21$

Data de publicação: 21/12/21

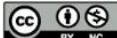

Esta obra está licenciada com uma licença

http://creativecommons.org/licenses/by-nc/4.0/

\section{RESUMO}

Introdução: Traumas na coluna vertebral podem trazer graves complicações. Relata-se caso de trauma em coluna cervicotorácica, com complicações comuns, tais como fratura de vértebras e hemopneumotórax bilateral. Entretanto, uma outra complicação rara destacou-se, o Hematoma Epidural Espinhal (HEE). Um acúmulo de sangue no espaço epidural que comprimiu mecanicamente a medula espinhal. Esta compressão comumente causa déficits neurológicos temporários ou permanentes. O tratamento mais efetivo é a descompressão cirúrgica, com evacuação do coágulo. Objetivo: Descrever um caso de HEE pós-traumático, com seu desfecho, através da análise de prontuário e estudos em literatura. Relato de caso: Relata-se caso de paciente de 59 anos, sexo masculino, com hematoma epidural espinhal pós-traumático da coluna torácica, com déficit neurológico tardio, paraplegia e anestesia ao nível de dermátomo T6. Realizada laminectomia descompressiva de T3-T4, seguida de flavectomia que evidenciou volumoso hematoma epidural espinhal, ocupando $2 / 3$ do canal medular. Após cirurgia houve melhora completa da anestesia, permanência da paraplegia, bem como perda de controle dos esfíncteres. Conclusão: O HEE é um evento raro, onde o diagnóstico precoce do hematoma e correção com a técnica de laminectomia descompressiva garantem ao paciente melhor prognóstico neurológico.

Palavras-chave: Traumatismos torácicos, Vértebras torácicas, Compressão de canal vertebral.

\section{ABSTRACT}

Introduction: Spinal trauma can lead to serious complications. We report a case of trauma to the cervicothoracic spine, with common complications, such as fractured vertebrae and bilateral hemopneumothorax. However, another rare complication stood out, the Spinal Epidural Hematoma (SHE). An accumulation of blood in the epidural space that mechanically compressed the spinal cord. This compression commonly causes temporary or permanent neurological deficits. The most effective treatment is surgical decompression, with evacuation of the clot. Objective: Describe a case of post-traumatic SHE, with its outcome, through the analysis of medical records and studies in the literature. Case-report: We report the case of a 59-year-old male patient with post-traumatic spinal epidural hematoma in the thoracic spine, with late neurological deficit, paraplegia and anesthesia at the T6 dermatome level. Decompressive T3-T4 laminectomy was performed, followed by a flavectomy that showed a large spinal epidural hematoma, occupying $2 / 3$ of the spinal canal. After surgery, there was complete improvement in anesthesia, persistence of paraplegia, as well as loss of control of the sphincters. Conclusion: SHE is a rare event, where early diagnosis of hematoma and correction with the decompressive laminectomy technique guarantees the patient a better neurological prognosis.

Keywords: Thoracic Injuries, Thoracic vertebrae, Spinal cord compression. 


\section{INTRODUÇÃO}

O Hematoma Epidural Espinhal (HEE) é uma condição rara, descrita pela primeira vez em 1869 por Jakso. A qual, pode ocorrer espontaneamente, por trauma na coluna, trauma por parto, discrasia sanguínea, terapia com anticoagulante, malformação arteriovenosa, anestesia ou punção lombar (KONG; MAK, 2003). Segundo Rechtine et al. (2001) o HEE é mais comumente associado a alterações cardiovasculares.

HEE pós-traumático, menos comum, aparece em 1 a 1,7\% de todas as lesões espinhais. $O$ tratamento mais usado é a descompressão cirúrgica de emergência (KONG; MAK, 2003).

Segundo Pear (1972) e Chan, Law e Chung (2006) a origem do sangramento é controversa. A teoria mais aceita é a de rupturas no plexo venoso do espaço epidural, decorrente de alteração abrupta da pressão venosa póstraumática. A hemorragia geralmente ocorre ao nível da medula espinhal com sintomas repentinos de dor focal e radicular, inicialmente, podendo desenvolver em horas, graus variáveis de paresia ou paralisia e alterações de sensibilidade.

O objetivo deste trabalho é divulgar um caso de HEE pós-traumático, com seu desfecho, através da análise de prontuário e estudos em literatura.

\section{RELATO DE CASO}

Artigo de Relato de caso escrito após submissão ao Comitê de Ética em Pesquisa (CEP), através da Plataforma Brasil. Parecer consubstanciado do CEP aprovado, em 11 de dezembro de 2020, sob o número de CAAE 39973120.0.0000.5246

Homem, 59 anos, dirigia um trator quando uma árvore caiu sobre ele. Deu entrada no pronto atendimento relatando dor em coluna cervicotorácica e dor pleurítica, ventilatório-dependente, ambas com melhora ao uso de analgésicos.

Realizada imobilização com colar cervical, estabilização clínica, evidenciando-se hemopneumotórax bilateral, tratado com drenagem em selo 
d'água. A tomografia computadorizada de coluna cervical evidenciou fratura horizontal completa da base do processo odontoide, sem desvios; Sinais de hiperostose esquelética idiopática difusa; Espondiloartrose cervical degenerativa; Protusões discais posteriores medianas nos níveis de C4-C5 a C6$\mathrm{C} 7$ com extensão foraminal à direita e esquerda, que reduz as respectivas bases foraminais e comprime as raízes emergentes de $\mathrm{C} 4$ a C6 bilateral. A tomografia de coluna torácica evidenciou fratura do processo transverso de T3 a direita, com desvios corticais; Espondilose dorsal; Fratura cominutiva no corpo vertebral de T4, acometendo predominantemente o aspecto superior do mesmo, com extensão para o platô vertebral superior e pedículo esquerdo.

Cerca de 2 dias após a admissão, o paciente apresentou parestesia em região dorsal e paraparesia. No dia seguinte evoluiu com perda de sensibilidade tátil, dolorosa e térmica ao nível do dermátomo T6, além de paraplegia. Em resposta ao parecer, neurocirurgião solicitou Ressonância Magnética por Imagem (RMI) de coluna vertebral, a qual fora realizada no município vizinho. Entre a solicitação do exame e a sua disponibilização decorreram cerca de 24 horas. A RMI de coluna torácica mostrou compressão extra-axial ao nível de T3 e T4, visível na Figura 1. 
Figura 1 - RMI sagital mostrando hematoma epidural espinhal

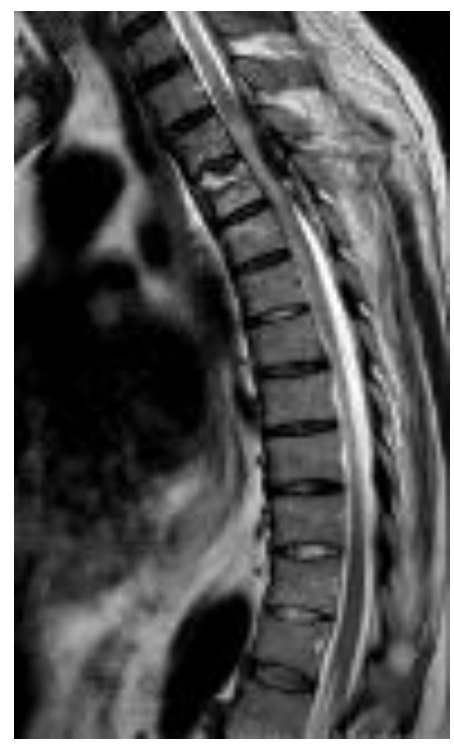

Fonte: arquivo pessoal do paciente

Realizada abordagem cirúrgica de urgência, laminectomia descompressiva ao nível de T3-T4, flavectomia seguido de visualização de volumoso hematoma epidural em canal medular ocupando os níveis de T3 e T4 com volume de aproximadamente $2 / 3$ do canal. Realizado também dexametasona $16 \mathrm{mg} /$ dia. É válido ressaltar que o paciente não fez uso de anticoagulantes, nem teve alterações no coagulograma. Recebeu alta da neurocirurgia 48h após procedimento cirúrgico, com melhora completa da anestesia e permanência da paraplegia; encaminhado para acompanhamento ambulatorial em uso de colar cervical e imobilização torácica, bem como perda de controle dos esfíncteres. Os drenos de tórax foram retirados 6 dias após internação, com boa evolução.

\section{DISCUSSÃO}

Alguns fatores de risco para o HEE pós-traumático são a espondilose cervical, a artrite reumatoide, a doença de Paget e a espondilite anquilosante (CUENCA et al., 2004). Neste relato de caso havia histórico de espondiloartrose degenerativa. 
Segundo Hansebout e Kachur (2021) as lesões traumáticas agudas de medula espinhal podem vir associadas a outras lesões cerebrais e sistêmicas tais como hemotórax, fraturas de extremidades e lesão intra-abdominal que podem limitar a capacidade de relatar dor localizada, complicando a avaliação inicial e o manejo destes pacientes, afetando o prognóstico. O paciente deste relato de caso teve, de forma inicial, a dor em região torácica, associado a hemopneumotórax bilateral que evoluiu, gradativamente, em dias, para a anestesia ao nível de T6, associado à paraplegia.

Em estudo de 11 hematomas, à radiologia, evidenciou-se que a Ressonância Magnética por Imagem é o exame de escolha para diagnóstico de HEE. Após 24h de acompanhamento, os hematomas apresentavam-se isointensos em relação à medula em imagens pesadas em $\mathrm{T} 1$ e heterogêneos em T2, produzindo tardiamente sinal alto tanto nas imagens em T1 como em T2. (BOUKOBZA et al, 1994). Todavia, segundo Hansebout e Kachur (2021), como a RMI nem sempre está disponível devido problemas de recursos e pessoal; em pacientes sem sinais de alerta; e em pacientes que forem observados movimentos motores grosseiramente normais em todas as extremidades, a tomografia computadorizada é suficiente nesta população. No caso em estudo, inicialmente, optou-se pela tomografia, pois o paciente preenchia os critérios acima citados. E quando o paciente apresentou paraparesia e paraplegia, respeitando os limites locais de disponibilidade tecnológica, a RMI foi realizada em município vizinho.

Segundo Chan, Law e Chung (2006); Segal, Lidov e Camins (1996); e Rodrigues et al (2010) a laminectomia descompressiva e evacuação do coágulo tem sido o tratamento mais utilizado, com melhora significativa dos sintomas. A cirurgia nas primeiras 12 horas é associada a melhor resultado neurológico (RODRIGUES, 2010). Num estudo retrospectivo de 32 casos concluiu-se que um atraso de mais de 36 horas para laminectomia descompressiva e evacuação do coágulo foi associado a um mau prognóstico (MCQUARRIE, 1978). Há relatos de melhora clínica com tratamento conservador a base de corticoides (JAMJOOM, 1996), o que justifica a administração de dexametasona no 
tratamento do paciente relatado. Seguindo a lógica desses estudos referidos, o paciente em questão, após evidenciado os sintomas neurológicos, seguiu processo de observação e solicitação de exames de imagem adequados ao quadro, com as melhores ferramentas e recursos disponíveis. Devido às dificuldades logísticas entre a realização do exame em Centro de imagem referenciado e o retorno do paciente ao hospital de origem decorreram-se 24 horas. Porém, após a avaliação da RMI, iniciaram-se os preparativos para a laminectomia de urgência, evoluindo com melhora da sensibilidade nos primeiros dias de pós-operatório. Não houve melhora da paraplegia e da perda de controle dos esfíncteres. Paciente permanece com quadro neurológico inalterado após um ano de acompanhamento ambulatorial.

\section{CONCLUSÃO}

Ao atender um paciente vítima de trauma raquimedular é importante lembrar das lesões mais comuns como fraturas de vértebras e extremidades, lesões intra-abdominais e hemopneumotórax. Todavia, é oportuno manter-se atento aos sinais e sintomas neurológicos que possam indicar uma condição mais rara como o HEE. Para pensar neste diagnóstico deve-se atentar aos fatores de risco para esta moléstia. A RMI, importante ferramenta diagnóstica na detecção precoce do HEE, associada a correção com a técnica de laminectomia descompressiva associam-se ao melhor prognóstico neurológico para o paciente. As lesões raquimedulares habitualmente causam déficits neurológicos. No caso em estudo houve alterações temporárias de sensibilidade, permanência da paraplegia, bem como perda de controle dos esfíncteres. 


\section{REFERÊNCIAS}

BOUKOBZA, M. et al. Spinal epidural haematoma: report of 11 cases and review of the literature. Neuroradiology, v. 36, n. 6, p. 456-459, 1994.

CHAN, K. M.; LAW, K. L.; CHUNG, C. H. Case Report: Thoracic Spinal Epidural Haematoma-an Unusual Cause of Chest Pain. Hong Kong Journal of Emergency Medicine, v. 13, n. 4, p. 217-220, 2006.

CUENCA, P.J. et al. Delayed traumatic spinal epidural hematoma with spontaneous resolution of symptoms. The Journal of emergency medicine, $v$. 27, n. 1, p. 37-41, 2004.

HANSEBOUT R.R.; KACHUR E. Acute traumatic spinal cord injury. In: AMINOFF, M.J.; MOREIRA, M.E.; WILTERDINK, J.L. (Ed.), UpToDate, 2021. Disponível em: https://www.uptodate.com/contents/acute-traumatic-spinal-cordinjury/contributors. Acessado em: 13 nov. 2021.

JAMJOOM, Z. A. Acute spontaneous spinal epidural hematoma: the influence of magnetic resonance imaging on diagnosis and treatment. Surgical neurology, v. 46, n. 4, p. 345-349, 1996.

KONG, J. K. F.; MAK, K. H. Spontaneous spinal epidural haematoma - An unusual cause of spinal cord compression. 2003.

MCQUARRIE, I.G. Recovery from paraplegia caused by spontaneous spinal epidural hematoma. Neurology, v. 28, n. 3, p. 224-224, 1978.

PEAR B.L. Spinal epidural hematoma. AJR, 115: 155-64, 1972.

RECHTINE, G.R. et al. Spontaneous resolution of symptomatic post-traumatic cervical epidural hematoma: a case report. JBJS, v. 83, n. 2, p. 255, 2001.

RODRIGUES, L.M.R. et al. Hematoma epidural pós-traumático tardio com evolução para déficit neurológico. Einstein (São Paulo), v. 8, p. 477-479, 2010.

SEGAL, D.H.; LIDOV, M.W.; CAMINS, M.B. Cervical epidural hematoma after chiropractic manipulation in a healthy young woman. Neurosurgery, v. 39, n. 5, p. 1043-1045, 1996. 